Supplement of Atmos. Chem. Phys., 17, 2839-2864, 2017

http://www.atmos-chem-phys.net/17/2839/2017/

doi:10.5194/acp-17-2839-2017-supplement

(C) Author(s) 2017. CC Attribution 3.0 License.

(c) (i)
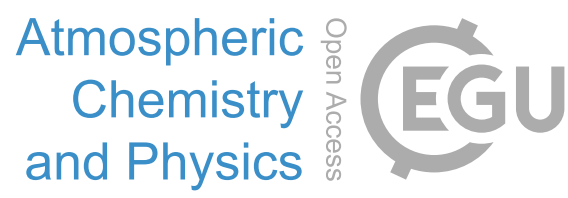

Supplement of

\title{
A comprehensive biomass burning emission inventory with high spatial and temporal resolution in China
}

Ying Zhou et al.

Correspondence to: Ying Zhou (y.zhou@bjut.edu.cn), Jianlei Lang (jllang@bjut.edu.cn) and Shuiyuan Cheng (chengsy@bjut.edu.cn)

The copyright of individual parts of the supplement might differ from the CC-BY 3.0 licence. 


\section{Supporting Information}

Section S1: Figure S1 The correlation between crop yield and grain yield at prefecture resolution.

Section S2: Figure S2 Maps showing the prefecture and county resolution.

Section S3: The details about questionnaire field survey.

Section S4: The detailed description about the MODIS fire data and calculation method and equation of gridded emission

Section S5: Figure S3 Daily $\mathrm{PM}_{2.5}$ biomass burning emissions variation in 2012.

Section S6: Figure S4 Emission of $\mathrm{PM}_{2.5}$ species from biomass burning.

Section S7: Figure S5 Emission of NMVOC species from biomass burning

Section S8: Table S1 CV (coefficients of variation) of biomass domestic burning emission factors.

Section S9: Table S2 CV (coefficients of variation) of biomass open burning emission factors 
S1 The correlation between crop yield and grain yield at prefecture resolution.

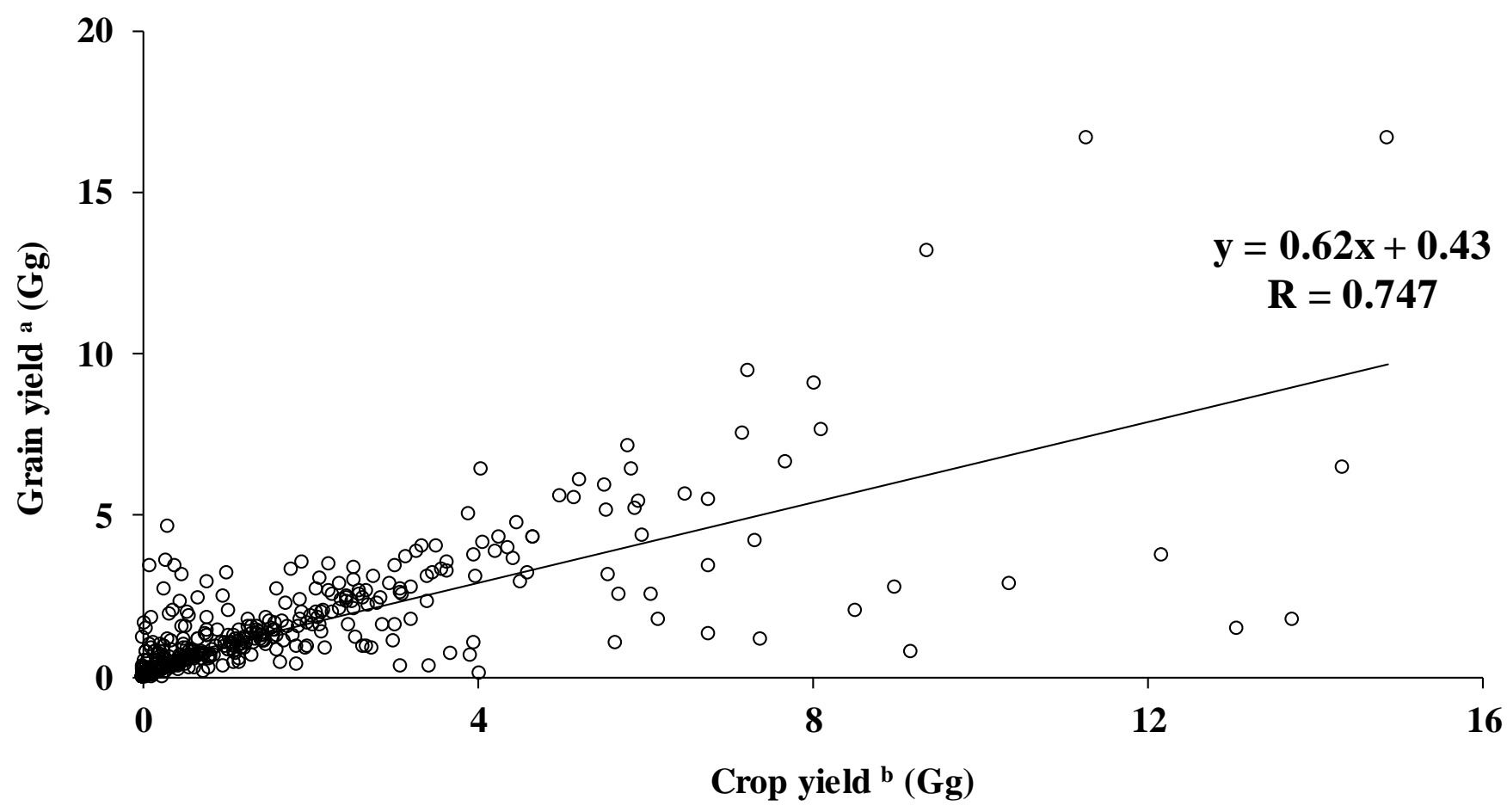

Figure S1 The correlation between crop yield and grain yield at prefecture resolution.

Note: ${ }^{a}$ NBSC (2013); ${ }^{b}$ a range of statistical yearbooks edited by National Bureau of Statistics in 2012 for each province. 
S2 Maps showing the prefecture and county resolution.

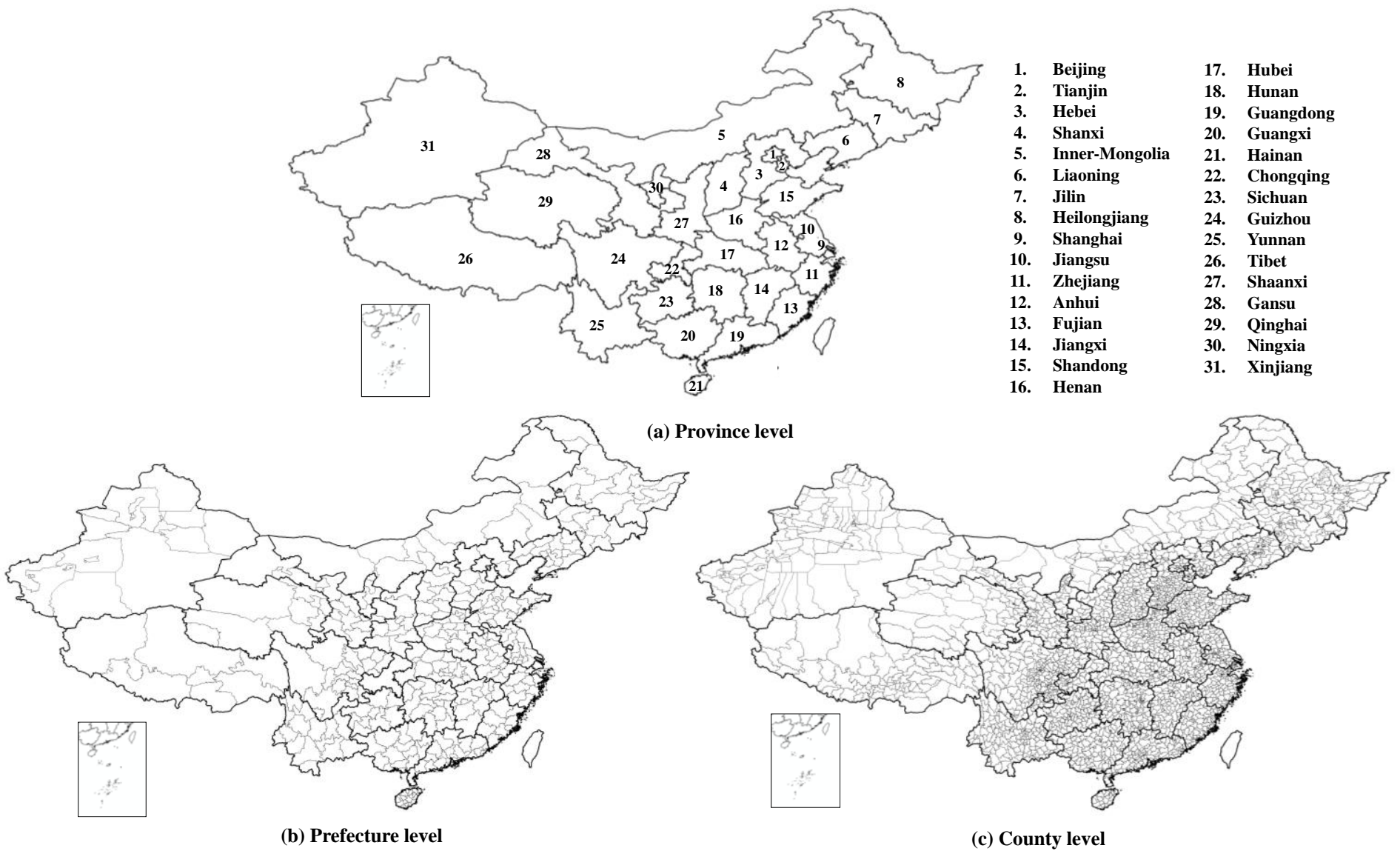

Figure S2 Maps showing the prefecture and county resolution. 


\section{S3 The details about questionnaire field survey.}

A questionnaire was designed to conduct field investigation during face-to-face interviews with rural resident, in order to obtain the percentage of domestic and in-field straw burning and uneven temporal distribution coefficient in several provinces with limited literature reports, including Tianjin, Hebei, Inner Mongolia, Heilongjiang, Shanghai, Zhejiang, Anhui, Jiangxi and Guangdong provinces. Respondents need to provide the detailed address, main cultivated crop type. They selected from a list of cooking and heating fuels, including specific crop straw, firewood, coal, gas, electricity or solar, livestock excrement and other detailed fuels not existing in the list. They also need to provide approximate proportion of crop straw domestic combustion and in field burning, and selected the month of burning the straw as waste, and heating period. The investigation was launched in the representative regions in each province mentioned above, with the integrative consideration about the geographical location, economic development level and population intensity. All the surveyors were trained and tested in their understanding of the questionnaire content. Ultimately, we received 2478 valid questionnaire responses, and at least 200 valid questionnaires in each province. 
S4 The detailed description about the MODIS fire data and calculation method and equation of gridded emission

\subsection{Detailed description about the MODIS fire data}

For the spatiotemporal distributions of biomass open burning, satellite remote sensing has excellent characteristics of wide coverage, high resolution and strong temporal reliability. As a result, satellite remote sensing has been increasingly applied to solving temporal and spatial emission distributions in recent years. The MODIS satellite fire data were taken from FIRM (Fire Information for Resource Management System). The MODIS Thermal Anomalies/Fire 5-Min L2 Swath Product (MOD14/MYD14) within $1 \mathrm{~km}$ resolution was used in this study. The MOD14 were provided by the Terra satellite with overpass times at 10:30 AM and 10:30 PM local time, while MYD14 were provided by Aqua at 1:30 AM and 1:30 PM local time.

\subsection{Detailed calculation method and equation of gridded emission}

The mass of biomass emission in each grid of biomass open burning and domestic burning was calculated using Eqs. (1) and (2), respectively, as follows:

$E_{m-o p e n}=\frac{F C_{m}}{F C_{n}} \times E_{n-o p e n}$

$E_{m-\text { domestic }}=\frac{P O_{m}}{P O_{n}} \times E_{n-\text { domestic }}$

where $m$ is the $m$-th grid and $n$ represents the $n$-th county; $\mathrm{E}_{m \text {-open }}$ and $\mathrm{E}_{n \text {-open }}$ represent the emissions of the $m$-th grid and $n$-th county for biomass open burning (in-field straw burning), respectively; $\mathrm{E}_{m \text {-domestic }}$ and $\mathrm{E}_{n \text {-domestic }}$ represent the emissions of the $m$-th grid and $n$-th county for biomass domestic burning, respectively; $\mathrm{FC}_{m}$ represents the number of typical fire points of the $m$-th grid; $\mathrm{FC}_{n}$ is the number of total typical fire points of the $n$-th county; $\mathrm{PO}_{m}$ is the number of typical population of the $m$-th grid; finally, $\mathrm{PO}_{n}$ is the number of typical population of the $n$-th county. 
S5 Daily PM2.5 biomass burning emissions variation in 2012.

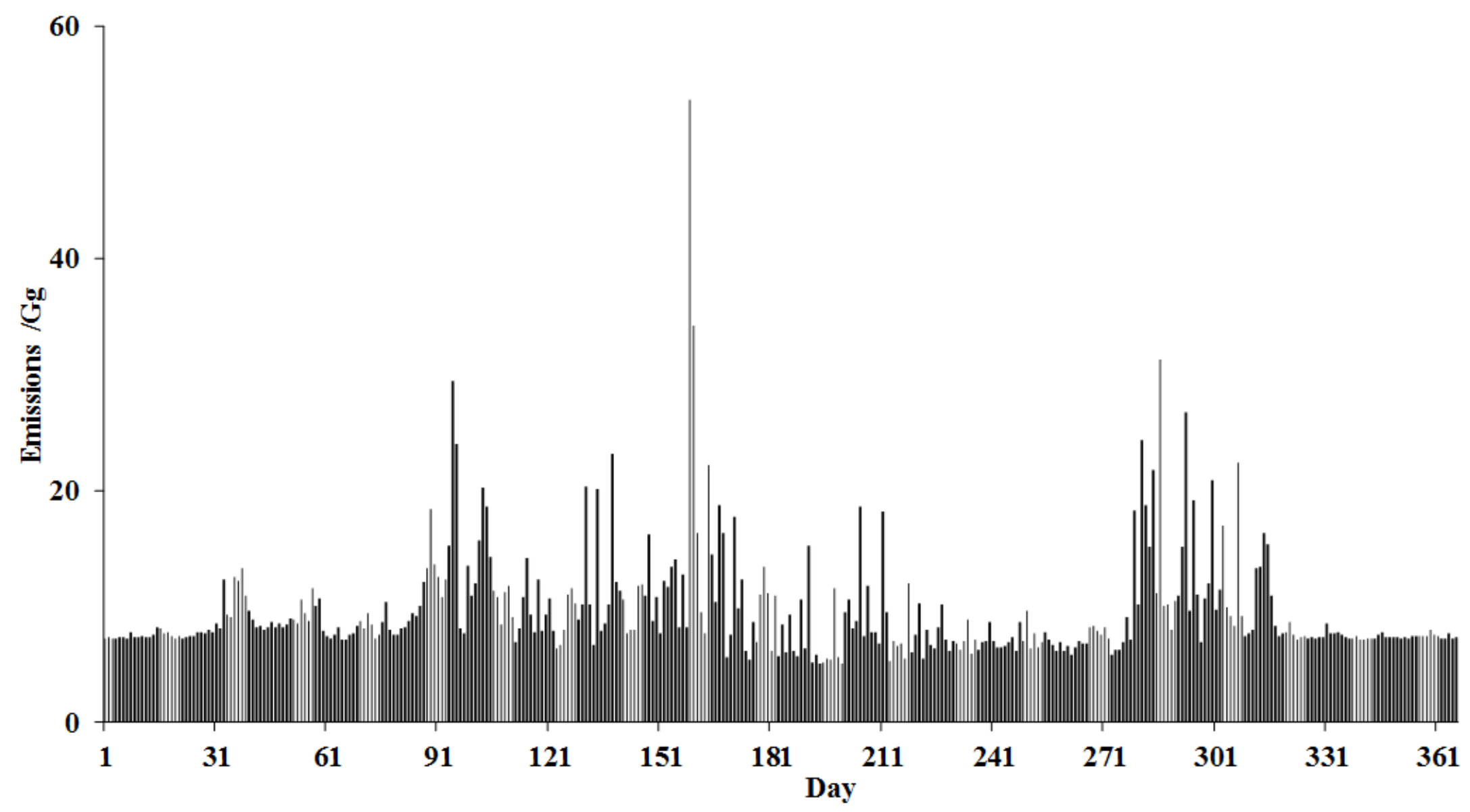

Figure S3 Daily PM2.5 biomass burning emissions variation in 2012. 


\section{S6 Emission of PM2.5 species from biomass burning}

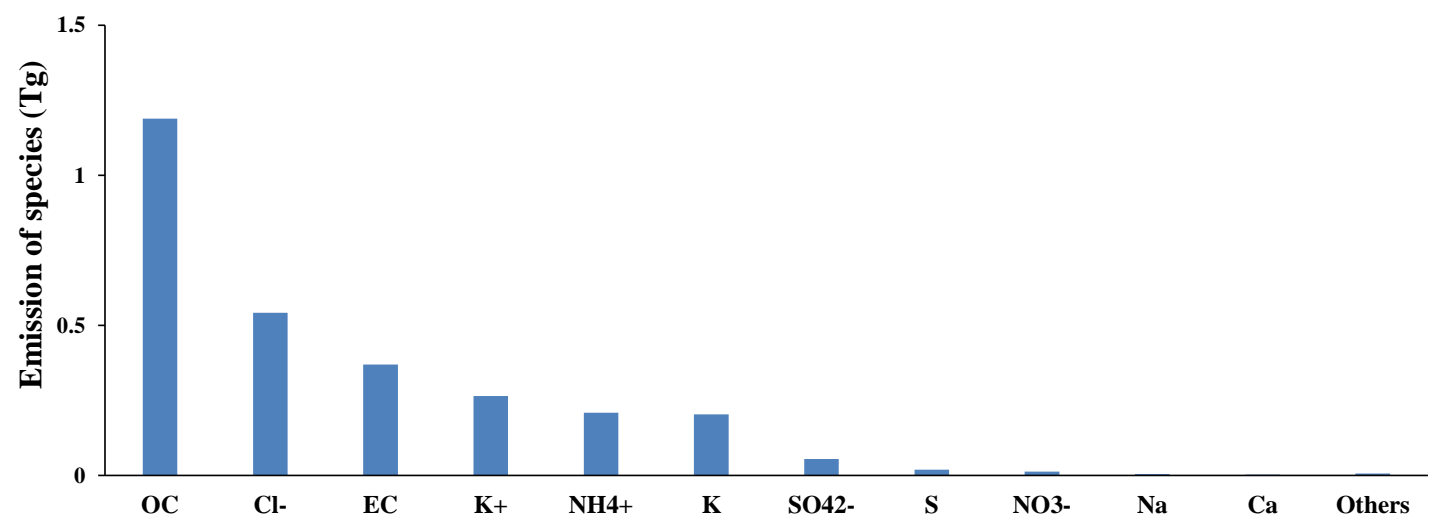

Figure S4 Emission of PM2.5 species from biomass burning.

Note: Species in others include $\mathrm{Al}, \mathrm{Si}, \mathrm{Mg}, \mathrm{Fe}, \mathrm{Pb}, \mathrm{Zn}, \mathrm{Ba}, \mathrm{Ti}, \mathrm{Ni}, \mathrm{Cr}, \mathrm{Mn}, \mathrm{Sr}, \mathrm{V}, \mathrm{Cd}, \mathrm{As}, \mathrm{Zr}$, Se, $\mathrm{Ag}, \mathrm{Sb}$, Sc, Mo, Ga, Tl, Co and Hg. $\mathrm{PM}_{2.5}$ speciation profile is obtained from Li et al., (2007) and Waston et al., (2001). 
S7 Emission of NMVOC species from biomass burning.

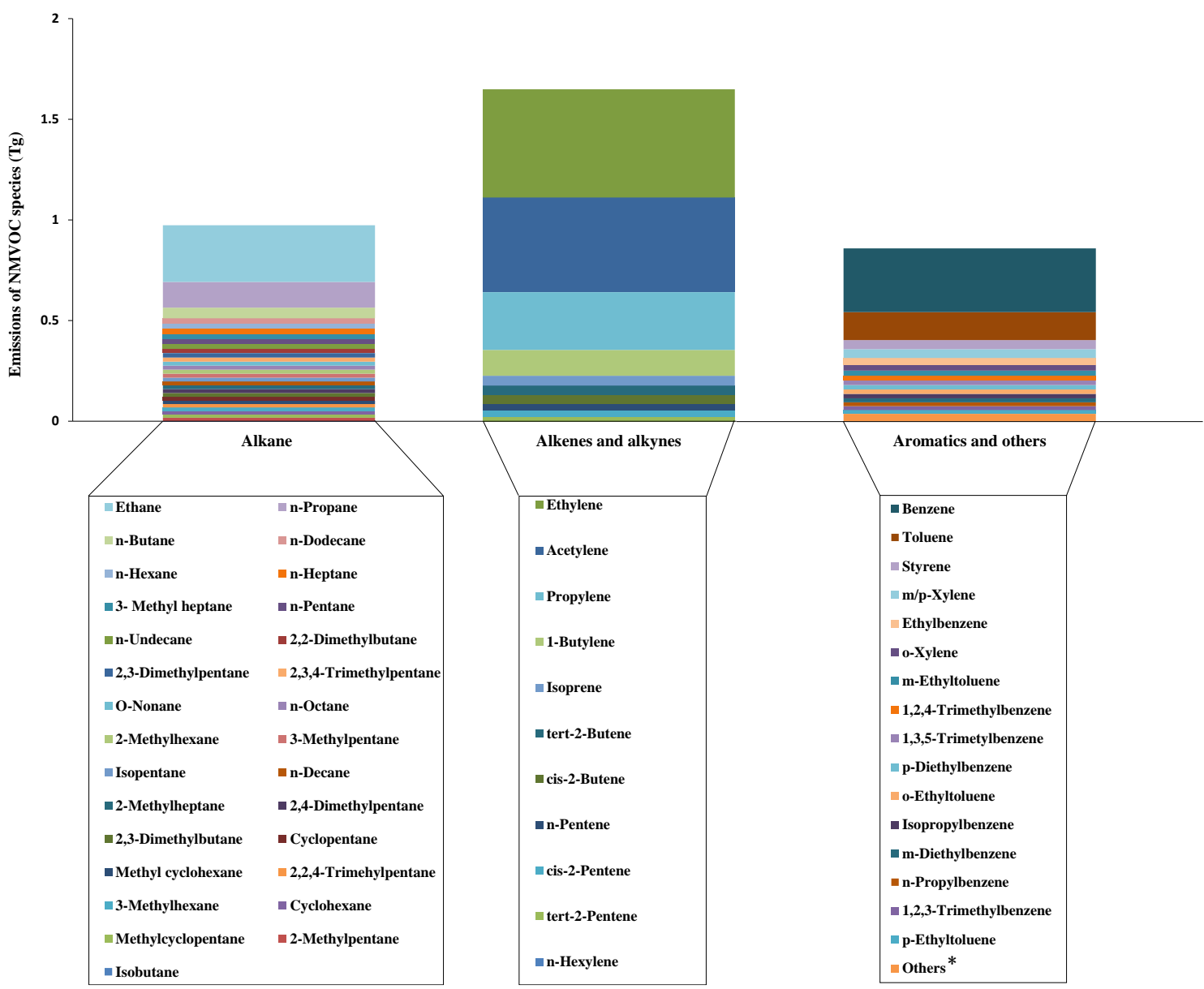

Figure S5 Emission of NMVOC species from biomass burning.

Note: *Species in others include aldehyde, ethers, alcohols, esters, ketone and acids. 
S8 Table S1 CV (coefficients of variation) of biomass domestic burning emission factors.

\begin{tabular}{|c|c|c|c|c|c|c|c|c|c|c|c|c|c|}
\hline & Material & $\mathrm{SO}_{2}$ & $\mathrm{NO}_{\mathrm{x}}$ & $\mathrm{PM}_{10}$ & $\mathrm{PM}_{2.5}$ & NMVOC & $\mathrm{NH}_{3}$ & $\mathrm{CO}$ & EC & OC & $\mathrm{CO}_{2}$ & $\mathrm{CH}_{4}$ & $\mathrm{Hg}$ \\
\hline \multirow{14}{*}{ 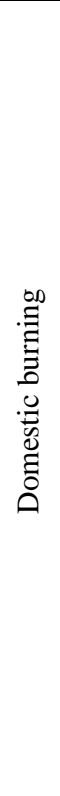 } & Corn & $0.5^{*}$ & $0.02^{\mathrm{a}}$ & $0.5^{*}$ & $0.27^{\mathrm{b}}$ & $0.5^{*}$ & $0.5^{*}$ & $0.85^{\mathrm{a}}$ & $0.34^{\mathrm{b}}$ & $0.44^{\mathrm{b}}$ & $0.04^{\mathrm{a}}$ & $0.5^{*}$ & $0.05^{\mathrm{c}}$ \\
\hline & Wheat & $0.5^{*}$ & $0.16^{\mathrm{a}}$ & $0.5^{*}$ & $0.23^{\mathrm{b}}$ & $0.5^{*}$ & $0.5^{*}$ & $0.89^{\mathrm{a}}$ & $0.76^{\mathrm{b}}$ & $0.29^{\mathrm{b}}$ & $0.07^{\mathrm{a}}$ & $0.5^{*}$ & $0.12^{\mathrm{c}}$ \\
\hline & Cotton & $0.5^{*}$ & $0.5^{*}$ & $0.5^{*}$ & $0.26^{\mathrm{b}}$ & $0.5^{*}$ & $0.5^{*}$ & $0.5^{*}$ & $0.39^{\mathrm{b}}$ & $0.55^{\mathrm{b}}$ & $0.5^{*}$ & $0.5^{*}$ & $0.33^{\mathrm{c}}$ \\
\hline & Cane & $0.5^{*}$ & $0.5^{*}$ & $0.5^{*}$ & $0.26^{\mathrm{b}}$ & $0.5^{*}$ & $0.5^{*}$ & $0.5^{*}$ & $0.63^{\mathrm{b}}$ & $0.45^{\mathrm{b}}$ & $0.5^{*}$ & $0.5^{*}$ & $0.32^{\mathrm{c}}$ \\
\hline & Potato & $0.5^{*}$ & $0.5^{*}$ & $0.5^{*}$ & $0.26^{\mathrm{b}}$ & $0.5^{*}$ & $0.5^{*}$ & $0.5^{*}$ & $0.63^{\mathrm{b}}$ & $0.45^{\mathrm{b}}$ & $0.5^{*}$ & $0.5^{*}$ & $0.53^{\mathrm{c}}$ \\
\hline & Peanut & $0.5^{*}$ & $0.5^{*}$ & $0.5^{*}$ & $0.26^{\mathrm{b}}$ & $0.5^{*}$ & $0.5^{*}$ & $0.5^{*}$ & $0.63^{\mathrm{b}}$ & $0.45^{\mathrm{b}}$ & $0.5^{*}$ & $0.5^{*}$ & $0.03^{c}$ \\
\hline & Rape & $0.5^{*}$ & $1.21^{\mathrm{d}}$ & $0.5^{*}$ & $0.15^{\mathrm{b}}$ & $0.26^{\mathrm{d}}$ & $0.5^{*}$ & $0.26^{\mathrm{d}}$ & $0.63^{\mathrm{b}}$ & $0.45^{\mathrm{b}}$ & $0.5^{*}$ & $0.5^{*}$ & $0.3^{\mathrm{c}}$ \\
\hline & Sesame & $0.5^{*}$ & $1.78^{\mathrm{d}}$ & $0.5^{*}$ & $0.26^{\mathrm{b}}$ & $0.24^{\mathrm{d}}$ & $0.5^{*}$ & $0.29^{\mathrm{d}}$ & $0.63^{\mathrm{b}}$ & $0.45^{\mathrm{b}}$ & $0.5^{*}$ & $0.5^{*}$ & $0.3^{\mathrm{c}}$ \\
\hline & Beet & $0.5^{*}$ & $0.5^{*}$ & $0.5^{*}$ & $0.26^{\mathrm{b}}$ & $0.5^{*}$ & $0.5^{*}$ & $0.5^{*}$ & $0.63^{b}$ & $0.45^{\mathrm{b}}$ & $0.5^{*}$ & $0.5^{*}$ & $0.3^{\mathrm{c}}$ \\
\hline & Hemp & $0.5^{*}$ & $0.5^{*}$ & $0.5^{*}$ & $0.26^{\mathrm{b}}$ & $0.5^{*}$ & $0.5^{*}$ & $0.5^{*}$ & $0.63^{\mathrm{b}}$ & $0.45^{\mathrm{b}}$ & $0.5^{*}$ & $0.5^{*}$ & $0.3^{c}$ \\
\hline & Rice & $0.5^{*}$ & $0.05^{\mathrm{a}}$ & $0.5^{*}$ & $0.29^{\mathrm{b}}$ & $0.5^{*}$ & $0.5^{*}$ & $0.06^{\mathrm{a}}$ & $0.65^{\mathrm{b}}$ & $0.5^{\mathrm{b}}$ & $0.01^{\mathrm{a}}$ & $0.5^{*}$ & $0.46^{\mathrm{c}}$ \\
\hline & Soybean & $0.5^{*}$ & $1.78^{\mathrm{d}}$ & $0.5^{*}$ & $0.26^{\mathrm{b}}$ & $0.76^{\mathrm{d}}$ & $0.5^{*}$ & $0.44^{\mathrm{d}}$ & $0.63^{\mathrm{b}}$ & $0.45^{\mathrm{b}}$ & $0.5^{*}$ & $0.5^{*}$ & $0.74^{c}$ \\
\hline & Firewood & $0.5^{*}$ & $1.42^{\mathrm{d}}$ & $0.5^{*}$ & $0.16^{\mathrm{b}}$ & $0.15^{\mathrm{d}}$ & $0.5^{*}$ & $0.39^{\mathrm{d}}$ & $0.46^{\mathrm{b}}$ & $0.35^{\mathrm{b}}$ & $0.5^{*}$ & $0.5^{*}$ & $1.17^{\mathrm{c}}$ \\
\hline & Feces & $0.8^{*}$ & $0.8^{*}$ & $0.8^{*}$ & $0.8^{*}$ & $0.8^{*}$ & $0.8^{*}$ & $0.8^{*}$ & $0.8^{*}$ & $0.8^{*}$ & $0.8^{*}$ & $0.8^{*}$ & $0.8^{*}$ \\
\hline
\end{tabular}

Note: Lowercase letters indicate the data source.

Sources are from the following: ${ }^{\text {a }}$ hang et al. (2008). ${ }^{\mathrm{b}}$ Li et al. (2009). ${ }^{\mathrm{c}}$ Chen et al. (2013). ${ }^{\mathrm{d}}$ Zhang et al. (2013). * Expert judgment data from Wei et al. (2011). 
S9 Table S2 CV (coefficients of variation) of biomass open burning emission factors

\begin{tabular}{|c|c|c|c|c|c|c|c|c|c|c|c|c|c|}
\hline & Material & $\mathrm{SO}_{2}$ & $\mathrm{NO}_{\mathrm{x}}$ & $\mathrm{PM}_{10}$ & $\mathrm{PM}_{2.5}$ & NMVOC & $\mathrm{NH}_{3}$ & $\mathrm{CO}$ & $\mathrm{EC}$ & $\mathrm{OC}$ & $\mathrm{CO}_{2}$ & $\mathrm{CH}_{4}$ & $\mathrm{Hg}$ \\
\hline \multirow{22}{*}{ 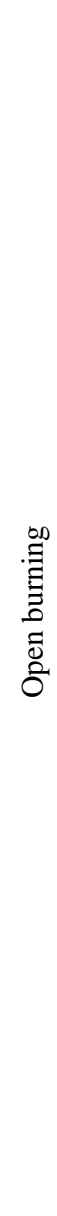 } & Corn & $0.45^{\mathrm{b}}$ & $0.42^{\mathrm{b}}$ & $0.5^{*}$ & $0.09^{\mathrm{b}}$ & $0.53^{b}$ & $0.76^{\mathrm{b}}$ & $0.08^{\mathrm{b}}$ & $0.33^{\mathrm{b}}$ & $0.39^{\mathrm{b}}$ & $0.01^{\mathrm{b}}$ & $0.22^{\mathrm{b}}$ & $0.05^{\mathrm{a}}$ \\
\hline & Wheat & $0.67^{\mathrm{b}}$ & $0.52^{\mathrm{b}}$ & $0.5^{*}$ & $0.54^{\mathrm{b}}$ & $0.25^{\mathrm{b}}$ & $0.38^{\mathrm{b}}$ & $0.41^{\mathrm{b}}$ & $0.32^{\mathrm{b}}$ & $0.26^{\mathrm{b}}$ & $0.03^{\mathrm{b}}$ & $0.25^{\mathrm{b}}$ & $0.12^{\mathrm{a}}$ \\
\hline & Cotton & $0.5^{*}$ & $0.5^{*}$ & $0.5^{*}$ & $0.5^{*}$ & $0.5^{*}$ & $0.5^{*}$ & $0.5^{*}$ & $0.5^{*}$ & $0.5^{*}$ & $0.5^{*}$ & $0.5^{*}$ & $0.33^{\mathrm{a}}$ \\
\hline & Cane & $0.5^{*}$ & $0.32^{\mathrm{d}}$ & $0.19^{\mathrm{d}}$ & $0.16^{\mathrm{d}}$ & $0.71^{\mathrm{d}}$ & $0.5^{*}$ & $0.61^{\mathrm{d}}$ & $1.57^{\mathrm{d}}$ & $0.2^{\mathrm{d}}$ & $0.18^{\mathrm{d}}$ & $0.5^{*}$ & $0.32^{\mathrm{a}}$ \\
\hline & Potato & $0.5^{*}$ & $0.5^{*}$ & $0.5^{*}$ & $0.5^{*}$ & $0.5^{*}$ & $0.5^{*}$ & $0.5^{*}$ & $0.5^{*}$ & $0.5^{*}$ & $0.5^{*}$ & $0.5^{*}$ & $0.53^{\mathrm{a}}$ \\
\hline & Peanut & $0.5^{*}$ & $0.5^{*}$ & $0.5^{*}$ & $0.5^{*}$ & $0.5^{*}$ & $0.5^{*}$ & $0.5^{*}$ & $0.5^{*}$ & $0.5^{*}$ & $0.5^{*}$ & $0.5^{*}$ & $0.03^{\mathrm{a}}$ \\
\hline & Rape & $0.5^{*}$ & $0.5^{*}$ & $0.5^{*}$ & $0.5^{*}$ & $0.5^{*}$ & $0.5^{*}$ & $0.5^{*}$ & $0.5^{*}$ & $0.5^{*}$ & $0.5^{*}$ & $0.5^{*}$ & $0.3^{\mathrm{a}}$ \\
\hline & Sesame & $0.5^{*}$ & $0.5^{*}$ & $0.5^{*}$ & $0.5^{*}$ & $0.5^{*}$ & $0.5^{*}$ & $0.5^{*}$ & $0.5^{*}$ & $0.5^{*}$ & $0.5^{*}$ & $0.5^{*}$ & $0.3^{\mathrm{a}}$ \\
\hline & Beet & $0.5^{*}$ & $0.5^{*}$ & $0.5^{*}$ & $0.5^{*}$ & $0.5^{*}$ & $0.5^{*}$ & $0.5^{*}$ & $0.5^{*}$ & $0.5^{*}$ & $0.5^{*}$ & $0.5^{*}$ & $0.3^{\mathrm{a}}$ \\
\hline & Hemp & $0.5^{*}$ & $0.5^{*}$ & $0.5^{*}$ & $0.5^{*}$ & $0.5^{*}$ & $0.5^{*}$ & $0.5^{*}$ & $0.5^{*}$ & $0.5^{*}$ & $0.5^{*}$ & $0.5^{*}$ & $0.3^{\mathrm{a}}$ \\
\hline & Rice & $0.5^{*}$ & $0.8^{\mathrm{d}}$ & $0.88^{\mathrm{d}}$ & $0.17^{\mathrm{d}}$ & $0.75^{\mathrm{d}}$ & $0.5^{*}$ & $1.19^{\mathrm{d}}$ & $1.38^{\mathrm{d}}$ & $1.53^{\mathrm{d}}$ & $0.14^{\mathrm{d}}$ & $0.5^{*}$ & $0.46^{\mathrm{a}}$ \\
\hline & Soybean & $0.5^{*}$ & $0.5^{*}$ & $0.5^{*}$ & $0.5^{*}$ & $0.5^{*}$ & $0.5^{*}$ & $0.5^{*}$ & $0.5^{*}$ & $0.5^{*}$ & $0.5^{*}$ & $0.5^{*}$ & $0.74^{\mathrm{a}}$ \\
\hline & Evergreen Needleleaf Forest & $0.3^{\mathrm{c}}$ & $0.39^{c}$ & $0.25^{\mathrm{d}}$ & $0.25^{\mathrm{d}}$ & $0.31^{\mathrm{e}}$ & $0.66^{\mathrm{e}}$ & $0.38^{\mathrm{e}}$ & $1^{\mathrm{f}}$ & $0.62^{\mathrm{f}}$ & $0.08^{\mathrm{e}}$ & $0.52^{\mathrm{e}}$ & $0.52^{g}$ \\
\hline & Evergreen Broadleaf Forest & $0.4^{\mathrm{e}}$ & $0.54^{\mathrm{e}}$ & $0.25^{\mathrm{d}}$ & $0.25^{\mathrm{d}}$ & - & $1.58^{\mathrm{h}}$ & $0.29^{\mathrm{e}}$ & $0.6^{\mathrm{e}}$ & $0.57^{\mathrm{e}}$ & $0.04^{\mathrm{e}}$ & $0.39^{\mathrm{e}}$ & $0.52^{\mathrm{g}}$ \\
\hline & Deciduous Needleleaf Forest & $0.3^{\mathrm{c}}$ & $0.23^{\mathrm{c}}$ & $0.25^{\mathrm{d}}$ & $0.25^{\mathrm{d}}$ & $0.31^{\mathrm{e}}$ & $0.66^{\mathrm{e}}$ & $0.38^{\mathrm{r}}$ & $1^{\mathrm{f}}$ & $0.62^{\mathrm{f}}$ & $0.08^{\mathrm{e}}$ & $0.52^{\mathrm{r}}$ & $0.52^{\mathrm{g}}$ \\
\hline & Deciduous Broadleaf Forest & $0.3^{\mathrm{c}}$ & $0.46^{\mathrm{e}}$ & $0.25^{\mathrm{d}}$ & $0.25^{\mathrm{d}}$ & $0.79^{\mathrm{e}}$ & $0.27^{\mathrm{e}}$ & $0.19^{\mathrm{e}}$ & $0.33^{\mathrm{e}}$ & $0.52^{\mathrm{e}}$ & $0.02^{\mathrm{e}}$ & $0.18^{\mathrm{e}}$ & $0.52^{\mathrm{g}}$ \\
\hline & Mixed Forest & $0.3^{\mathrm{c}}$ & $0.46^{\mathrm{e}}$ & $0.25^{\mathrm{d}}$ & $0.25^{\mathrm{d}}$ & $0.62^{\mathrm{e}}$ & $0.27^{\mathrm{e}}$ & $0.19^{\mathrm{e}}$ & $0.33^{\mathrm{e}}$ & $0.52^{\mathrm{e}}$ & $0.02^{\mathrm{e}}$ & $0.18^{\mathrm{e}}$ & $0.52^{\mathrm{g}}$ \\
\hline & Closed Shrublands & $0.44^{\mathrm{e}}$ & $0.21^{\mathrm{e}}$ & $0.25^{\mathrm{d}}$ & $0.25^{\mathrm{d}}$ & $0.48^{\mathrm{e}}$ & $0.33^{\mathrm{e}}$ & $0.25^{\mathrm{e}}$ & $0.4^{\mathrm{f}}$ & $0.18^{\mathrm{f}}$ & $0.02^{\mathrm{e}}$ & $0.35^{\mathrm{e}}$ & $0.74^{\mathrm{h}, \mathrm{g}}$ \\
\hline & Open Shrublands & $0.44^{\mathrm{e}}$ & $0.21^{\mathrm{e}}$ & $0.25^{\mathrm{d}}$ & $0.25^{\mathrm{d}}$ & $0.48^{\mathrm{e}}$ & $0.33^{\mathrm{e}}$ & $0.25^{\mathrm{e}}$ & $0.4^{\mathrm{f}}$ & $0.18^{\mathrm{f}}$ & $0.02^{\mathrm{e}}$ & $0.35^{\mathrm{e}}$ & $0.74^{\mathrm{h}, \mathrm{g}}$ \\
\hline & Woody Savannas & $0.44^{\mathrm{e}}$ & $0.21^{\mathrm{e}}$ & $0.25^{\mathrm{d}}$ & $0.25^{\mathrm{d}}$ & $0.48^{\mathrm{e}}$ & $0.33^{\mathrm{e}}$ & $0.25^{\mathrm{e}}$ & $0.4^{\mathrm{f}}$ & $0.18^{\mathrm{f}}$ & $0.02^{\mathrm{e}}$ & $0.35^{\mathrm{e}}$ & $0.52^{\mathrm{h}}$ \\
\hline & Savannas & $0.63^{\mathrm{e}}$ & $0.29^{\mathrm{e}}$ & $0.25^{\mathrm{d}}$ & $0.25^{\mathrm{d}}$ & $0.25^{\mathrm{e}}$ & $0.8^{\mathrm{e}}$ & $0.29^{\mathrm{e}}$ & $0.5^{\mathrm{e}}$ & $0.46^{\mathrm{e}}$ & $0.02^{\mathrm{e}}$ & $0.6^{\mathrm{e}}$ & $0.52^{\mathrm{h}}$ \\
\hline & Grasslands & $0.63^{\mathrm{e}}$ & $0.29^{\mathrm{e}}$ & $0.25^{\mathrm{d}}$ & $0.25^{\mathrm{d}}$ & $0.25^{\mathrm{e}}$ & $0.8^{\mathrm{e}}$ & $0.29^{\mathrm{e}}$ & $0.5^{\mathrm{e}}$ & $0.46^{\mathrm{e}}$ & $0.02^{\mathrm{e}}$ & $0.6^{\mathrm{e}}$ & $0.52^{\mathrm{h}}$ \\
\hline
\end{tabular}

Note: Lowercase letters indicate the data source.

Sources are from the following: ${ }^{\mathrm{a}}$ Chen et al. (2013). ${ }^{\mathrm{b}}$ Li et al. (2007). ${ }^{\mathrm{c}}$ Andreae and Rosenfeld (2008). ${ }^{\mathrm{d}}$ Song et al. (2009). ${ }^{\mathrm{e}}$ Akagi et al. (2011). ${ }^{\mathrm{f}}$ McMeekin et al. (2008). ${ }^{\mathrm{g}}$ Friedli et al. (2003). ${ }^{\mathrm{h}}$ 
Streets et al. (2005). * Expert judgment data from Wei et al. (2011).

\section{Reference}

Akagi, S. K., Yokelson, R. J., Wiedinmyer, C., Alvarado, M. J., Reid, J. S., Karl, T., Crounse, J. D., and Wennberg, P. O.: Emission factors for open and domestic biomass burning for use in atmospheric models, Atmos. Chem. Phys., 11, 4039-4072, doi:10.5194/acp-11-4039-2011, 2011.

Andreae, M. O. and Rosenfeld, D.: Aerosol-cloud-precipitation interactions. Part 1, The nature and sources of cloud-active aerosols, Earth Sci. Rev., 89, 13-41, doi:10.1016/j.earscirev.2008.03.001, 2008.

Chen, C., Wang, H. H., Zhang, W., Hu, D., Chen, L. and Wang, X. J.: High-resolution inventory of mercury emissions from biomass burning in China for 2000-2010 and a projection for 2020, Journal of Geophysical Research-Atmospheres, 118, 12248-12256, doi: 10.1002/2013jd019734, 2013.

Friedli, H. R., Radke, L. F., Prescott, R., Hobbs, P. V., and Sinha, P., Mercury emissions from the August 2001 wildfires in Washington State and an agricultural waste fire in Oregon and atmospheric mercury budget estimates, Global Biogeochem. Cycle, 17(2), 1039, doi:10.1029/ 2002GB001972, 2003.

Li, X. H., Wang, S. X., Duan, L., Hao, J. M. and Nie, Y. F.: Carbonaceous Aerosol Emissions from Household Biofuel Combustion in China, Environ. Sci. Technol., 43, 6076-6081, doi: 10.1021/es803330j; 2009.

Li, X. H., Wang, S. X., Duan, L., Hao, J., Li, C., Chen, Y. S. and Yang, L.: Particulate and trace gas emissions from open burning of wheat straw and corn stover in China, Environ. Sci. Technol., 41, 6052-6058, doi: 10.1021/es0705137, 2007.

McMeeking, G. R.: The optical, chemical, and physical properties of aerosols and gases emitted by the laboratory combustion of wildland fuels, Ph.D. Dissertation, Department of Atmospheric Sciences, Colorado State University, 109-113, Fall 2008.

National Bureau of Statistics of China (NBSC): China Statistical Yearbook for Regional Economy 2013, China Statistics Press, Beijing, 2013, available at 
http://www.stats.gov.cn/tjsj/tjcbw/, (in Chinese).

Song, Y., Liu, B., Miao, W., Chang, D. and Zhang, Y.: Spatiotemporal variation in non-agricultural open fire emissions in China from 2000 to 2007 , Global Biogeochem.

Cycles, 23, GB2008, doi: 10.1029/2008GB003344, 2009.

Streets, D. G., Hao, J. M., Wu, Y., Jiang, J. K., Chan, M., Tian, H. Z. and Feng, X. B.: Anthropogenic mercury emissions in China, Atmos. Environ., 39, 7789-7806, doi: 10.1016/j.atmosenv.2005.08.029, 2005.

Watson, J. G., Chow, J. C. and Houck, J. E.: PM $_{2.5}$ chemical source profiles for vehicle exhaust, vegetative burning, geological material, and coal burning in Northwestern Colorado during 1995, Chemosphere, 43, 1141-1151, doi: 10.1016/s0045-6535(00)00171-5, 2001.

Wei, W., Wang, S. X. and Hao, J. M: Uncertainty Analysis of Emission Inventory for Volatile Organic Compounds from Anthropogenic Sources in China, Environmental Science, 32, 305-312, 2011 (in Chinese).

Zhang, H. F., Ye, X. N., Cheng, T. T., Chen, J. M., Yang, X., Wang, L. and Zhang, R. Y.: A laboratory study of agricultural crop residue combustion in China: Emission factors and emission inventory, Atmos. Environ. 42, 8432-8441, doi: 10.1016/j.atmosenv.2008.08.015, 2008.

Zhang, W., Wei, W., Hu, D., Zhu, Y. and Wane, X.J.: Emission of Speciated Mercury from Residential Biomass Fuel Combustion in China. Energy \& Fuels, 27, 6792-6800, doi: 10.1021/ef401564r, 2013. 